\title{
Arthroscopic capsular release versus manipulation under anesthesia for primary frozen shoulder
}

\author{
Jung-Taek Hwang \\ Department of Orthopedic Surgery, Chuncheon Sacred Heart Hospital, Hallym University Medical College, Chuncheon, Korea
}

Arthroscopic capsular release (ACR) and manipulation under anesthesia (MUA) are treatment options for primary frozen shoulder (FS). Both of these are useful for primary FS [1-3]. In addition, MUA is possible without the surgical equipment needed for an arthroscopic procedure. The indications for the two procedures are not different, either [1,2].

On this topic, a study by Lee et al. [4], "Can manipulation under anesthesia alone provide clinical outcomes similar to arthroscopic circumferential capsular release in primary frozen shoulder (FS)?: the necessity of arthroscopic capsular release in primary FS" retrospectively reviewed 54 patients treated with MUA and 22 patients treated with ACR. They compared the clinical outcome of both groups with one year follow up after the procedures, and reported that the outcome variables at 3 months after surgery and the improvement of outcome variables did not show any difference between both groups, but in the evaluation of pain and range of motion at 1 week, the MUA group showed significantly better results than the ACR group. They concluded that MUA alone can provide a similar clinical outcome as ACR in refractory FS.

Although MUA is an useful option for primary FS, several complications such as proximal humerus fracture, shoulder dislocation, brachial plexus stretching injury, rotator cuff injury, and glenoid fracture may result $[1-3,5]$. Therefore, MUA should be performed with great caution. They reported that 11 patients ( $12.2 \%$ in the MUA group and $18.2 \%$ in the ACR group) needed additional steroid injection between 8 to 16 weeks after surgery, and the necessity of additional injections was three times higher in diabetics compared to nondiabetics. Previous studies have reported that intraarticular steroid injection is a useful treatment option for primary FS in out-patient department [6-8]. However, additional steroid injection within the follow-up period could be a bias that compromises the reliability of this study.

In addition, a retrospective comparative study by Lee et al. [4] compared patients treated with MUA to patients treated with ACR that followed a limited MUA. The preceding limited MUA could be a source of bias, also. Therefore, the results of this study should be interpreted with caution. Another comparative study reported greater gain in range of motion in a MUA group compared to an ACR group[1]. According to a systemic review of 22 studies, which included 989 patients, there were minimal differences in shoulder range of motion or Constant score between MUA and ACR groups for treatment of refractory FS, and it concluded the data available demonstrate little benefit for ACR instead of, or in addition to, MUA [2]. Well-designed comparative studies are needed.

In my opinion, MUA and ACR are good treatment options for primary FS. MUA is more simple and easy than ACR because ar-

Received: November 11, 2020 Revised: November 17, 2020 Accepted: November 20, 2020

Correspondence to: Jung-Taek Hwang

Department of Orthopedic Surgery, Chuncheon Sacred Heart Hospital, Hallym University Medical College, 77 Sakju-ro, Chuncheon 24253, Korea

Tel: +82-33-240-5197, Fax: +82-33-252-0177, E-mail: drakehjt@hanmail.net, ORCID: https://orcid.org/0000-0003-4189-084X

Financial support: None.

Conflict of interest: None.

Copyright(C) 2020 Korean Shoulder and Elbow Society. All Rights Reserved.

This is an Open Access article distributed under the terms of the Creative Commons Attribution Non-Commercial License (http://creativecommons.org/licenses/by-nc/4.0/) which permits unrestricted non-commercial use, distribution, and reproduction in any medium, provided the original work is properly cited. 
throscopic equipment is not necessary. However, MUA can lead to several serious complications $[1-3,5]$. If experienced surgeons perform ACR, it can be more safe than MUA. Moreover, ACR is convenient for patients with rotator cuff tears combined with secondary FS [9]. Also, ACR followed by MUA could be better than MUA followed by ACR to avoid the mentioned complications seen with MUA.

\section{ORCID}

Jung-Taek Hwang

https://orcid.org/0000-0003-4189-084X

\section{REFERENCES}

1. Kim DH, Song KS, Min BW, Bae KC, Lim YJ, Cho CH. Early clinical outcomes of manipulation under anesthesia for refractory adhesive capsulitis: comparison with arthroscopic capsular release. Clin Orthop Surg 2020;12:217-23.

2. Grant JA, Schroeder N, Miller BS, Carpenter JE. Comparison of manipulation and arthroscopic capsular release for adhesive capsulitis: a systematic review. J Shoulder Elbow Surg 2013;22: 1135-45.

3. Yoo JC, Koh KH, Shon MS, Bae KH, Lim TK. Clinical outcome after arthroscopic capsular release for adhesive capsulitis of the shoulder. Clin Shoulder Elbow 2018;21:127-33.
4. Lee SJ, Jang JH, Hyun YS. Can manipulation under anesthesia alone provide clinical outcomes similar to arthroscopic circumferential capsular release in primary frozen shoulder (FS)?: the necessity of arthroscopic capsular release in primary FS. Clin Shoulder Elbow 2020;23:169-77.

5. Magnussen RA, Taylor DC. Glenoid fracture during manipulation under anesthesia for adhesive capsulitis: a case report. J Shoulder Elbow Surg 2011;20:e23-6.

6. Kim DY, Lee SS, Nomkhondorj O, et al. Comparison between anterior and posterior approaches for ultrasound-guided glenohumeral steroid injection in primary adhesive capsulitis: a randomized controlled trial. J Clin Rheumatol 2017;23:51-7.

7. Kim YS, Lee HJ, Lee DH, Choi KY. Comparison of high- and low-dose intra-articular triamcinolone acetonide injection for treatment of primary shoulder stiffness: a prospective randomized trial. J Shoulder Elbow Surg 2017;26:209-15.

8. Jacobs LG, Smith MG, Khan SA, Smith K, Joshi M. Manipulation or intra-articular steroids in the management of adhesive capsulitis of the shoulder? A prospective randomized trial. J Shoulder Elbow Surg 2009;18:348-53.

9. Cho $\mathrm{CH}$, Jang $\mathrm{HK}$, Bae $\mathrm{KC}$, et al. Clinical outcomes of rotator cuff repair with arthroscopic capsular release and manipulation for rotator cuff tear with stiffness: a matched-pair comparative study between patients with and without stiffness. Arthroscopy 2015;31:482-7. 